\title{
Migraine improvement correlates with posterior cingulate cortical thickness reduction
}

\author{
Variação da espessura cortical no cíngulo posterior em pacientes com enxaqueca. \\ Correlação com a melhora pós-tratamento profilático
}

\author{
Vanise C. G. Amaral', Gustavo Tukamoto², Tadeu Kubo², Ronir Raggio Luiz³, Emerson Gasparetto ${ }^{4}$, \\ Maurice B. Vincent 5,6
}

\begin{abstract}
Objective: The main goal of this study was to correlate migraine improvement, after prophylactic therapy, with cortical thickness changes. Methods: Cortical thickness maps were obtained with magnetic resonance imaging (MRI) from 19 patients with migraine before (first scan) and after (second scan) prophylactic treatment, and these were compared with controls using the FreeSurfer MRI tool. Cortical changes were correlated with the headache index (HI). Results: Anincrease incortical thickness was found in the right cuneus and precuneus, somatosensory and superior parietal cortices in both patient scans, compared with the controls. No changes were observed in the left hemisphere. Following correction for multiple comparisons, no areas changed from the first to the second scan. Regression analysis showed a significant negative correlation between the $\mathrm{HI}$ improvement and cortical thickness changes in the left posterior cingulate, a region involved with nociception and, possibly, the development of chronic pain. Conclusion: There were changes in cortical thickness in patients with migraine relative to controls in areas involved with vision and pain processing. Left posterior cingulate cortical changes correlated with headache frequency and intensity.
\end{abstract}

Keywords: migraine disorders; magnetic resonance imaging - FreeSurfer; prophylactic treatment.

\section{RESUMO}

Objetivos: Correlacionar a melhora de pacientes enxaquecosos após tratamento preventivo com alterações na espessura do córtex cerebral. Métodos: Espessura cortical foi determinada a partir de imagens de ressonância magnética (RM)em 19 pacientes com enxaqueca, antes (1 $1^{a}$ RM) e após (2a RM) o tratamento profilático, e comparada com controles, usando o programa FreeSurfer. Mudanças corticais foram correlacionadas com o índice de cefaleia (HI). Resultados: O hemisfério direito apresentou aumento da espessura no córtex do cúneus e pré-cúneus, parietal superior e somatossensitivo na primeira RM e na segunda RM, em comparação aos controles. Após correção para comparações múltiplas, nenhuma região cortical se mostrou estatisticamente diferente entre a primeira e a segunda RM. A regressão mostrou correlação (negativa) significativa entre melhora do HI e mudanças na espessura cortical do cíngulo posterior esquerdo. Conclusão: Existem alterações de espessura cortical em pacientes com enxaqueca em relação a controles em áreas envolvidas com processamento visual e com a dor. As alterações corticais no cíngulo posterior esquerdo variaram de acordo com a frequência e intensidade das crises.

Palavras-chave: enxaqueca; ressonância magnética - Free Surfer; tratamento profilático.

Migraine, the most prevalent neurological disease, affects mostly females at the peak of productivity in life ${ }^{1}$. It is characterized by paroxysmal intense unilateral, throbbing head pain attacks accompanied by varying combinations of hypersensitivity to light, sound and smell, nausea, vomiting, as well as a variety of autonomic, cognitive, emotional and motor disturbances ${ }^{2}$.
Variations in cortical thickness have been found in many physiological conditions and neurological disorders, including migraine ${ }^{3}$, but the relevance, reversibility, and nature of these changes remain controversial ${ }^{4,5}$. Among other findings, voxel-based morphometry and diffusion tensor imaging have revealed thickening of the somatosensory cortex ${ }^{3,4,6}$,

\footnotetext{
'Universidade Estadual do Amazonas, Departamento de Neurologia e Neurocirurgia, Manaus AM, Brasil;

${ }^{2}$ Clínica de Diagnóstico por Imagem (CDPI), Rio de Janeiro RJ, Brasil;

${ }^{3}$ Universidade Federal do Rio de Janeiro, Departamento de Saúde Pública, Rio de Janeiro RJ, Brasil;

«Universidade Federal do Rio de Janeiro, Hospital Universitário Clementino Fraga Filho, Departamento de Radiologia, Rio de Janeiro RJ, Brasil; ${ }^{5}$ Universidade Federal do Rio de Janeiro, Hospital Universitário Clementino Fraga Filho, Departamento de Neurologia, Rio de Janeiro RJ, Brasil; ${ }^{6}$ Neuroscience. Eli Lilly and Company, Indianapolis, USA.

Correspondence: Vanise C. G. Amaral; Universidade do Estado do Amazonas, Escola Superior de Ciências da Saúde; Av Carvalho Leal, 1777; 69065-130 Manaus AM, Brasil; E-mail: vaniseamaral@gmail.com

Conflict of interest: Maurice Vincent is a research fellow at Eli Lilly and Company. The other authors did not declare any conflict of interest.

Received 21 September 2017; Received in final form 29 November 2017; Accepted 09 December 2017.
} 
increased gray matter density in the caudate nucleus ${ }^{7}$ and gray matter volume loss in the superior temporal gyrus, inferior frontal gyrus, precentral gyrus, anterior cingulate cortex, amygdala, parietal operculum, middle and inferior frontal gyrus and bilateral insula ${ }^{6}$. Changes in cortical or subcortical structures depend on the frequency of migraine attacks for a number of cortical regions ${ }^{3,7}$. Functional imaging techniques used to measure relative activations in different brain areas point to a migraine generator in the brainstem, probably in the dorsal rostral pons ${ }^{8}$.

Available data suggest that some cerebral changes are associated with longer disease duration and increased migraine frequency. Migraine has a benign course and improves with age ${ }^{9}$. This contradicts possible cumulative migraine-related effects on brain structure.

It remains unclear whether the migraine-related structural changes are a consequence of repetitive attacks or a substratum that contributes to the migraine susceptibility. Few studies have focused on the reversibility of migraine-related cerebral changes. The main goal of this study was to correlate migraine improvement, after prophylactic therapy, with cortical thickness changes.

\section{METHODS}

\section{Participants}

This study was performed at the Headache Unit, Hospital Universitário Clementino Fraga Filho, Rio de Janeiro, Brazil, and CDPI Neuroimaging facility, Rio de Janeiro (Local ethics committee approval \#123/11 according to the Declaration of Helsinki).

Sixty-eight prophylaxis-naïve patients with episodic migraine with aura, migraine without aura, and chronic migraine (IHS-II ${ }^{10}$ and, retrospectively, IHS-III beta ${ }^{11}$ diagnostic criteria), successively selected either from the University Hospital headache outpatient unit or private practice (MV), were eligible to participate in this study. Patients with claustrophobia and/or any other known contraindication did not qualify. Seventeen patients were excluded due to technical errors in magnetic resonance imaging (MRI) data acquisition, 29 refused to be scanned or did not fulfill the inclusion criteria, and three were removed because of MRI post-processing technical concerns. From the original 25 historical headache-free participants selected at the CDPI for comparison, six were removed for optimal age and gender matching. A comprehensive neurological examination was performed to exclude concomitant systemic or neurological disorders.

Before any prophylactic treatment, subjects provided information about the average number of attacks per month for at least the last three months, attack duration, and severity as mild, moderate, intense or very intense according to their impact on daily activity. A headache index (HI) was calculated as the attack duration (hours) multiplied by the frequency of attacks in one month. Subjects underwent a first scan and, after image acquisition, routine prophylaxis (usual care according to our individualized routine procedures as for every new migraine patient without any drug preference) was prescribed. After a variable period of time $(1,893.8 \pm 555.3$ days, $\min =339 ; \max =2,247)$ patients were rescanned under identical protocols. The clinical variables and the HI were recorded again.

\section{MRI acquisition and analysis}

The MRI data were acquired on a 3 Tesla (T) scanner (Magnetom Trio Tim, Siemens, Erlangen, Germany), using a 12-channel head coil. The MRI protocol included: sagittal T1 3D magnetization prepared rapid gradient echo (MPRAGE) weighted image (TR, $2530 \mathrm{~ms}$; TE, $3.45 \mathrm{~ms}$; inversion time, 1100 ms; FOV, 256 mm; matrix, 256 x 256; 1.0 mm section thickness, flip angle, 7 ; voxel size, $1.3 \times 1.0 \times 1.3 \mathrm{~mm}$ ), The participants' heads were stabilized with tape across the forehead and padding around the sides. All MRIs were reviewed by experienced neuroradiologists and were of good quality for post-processing.

\section{Cortical thickness and statistics}

Cortical reconstruction and volumetric segmentation was performed using Free Surfer version 5.0.0 (http://surfer. nmr.mgh.harvard.edu) normalization of intensity; tessellation of the gray matter/white matter boundary; automated topology correction; surface deformation following intensity gradients to optimally place the gray matter/white matter and gray matter/CSF borders at the location where the greatest shift in intensity defines these transitions; and inflation of the brain.

This method used both intensity and continuity information from the entire three-dimensional MR volume in segmentation and deformation procedures to produce representations of cortical thickness, calculated as the closest distance from the gray matter/white matter boundary to the gray matter/CSF boundary at each vertex on the tessellated surface. Once an accurate white matter/gray matter surface had been created, the pial surface was generated by expanding the white matter surface, so that it closely followed the gray/CSF intensity gradient without crossing the white matter surface boundary. After the pial surface was generated, it was visually checked for defects that may have been created during automatic topology fixing. If the surface appeared to deviate from the gray matter/CSF boundary, manual editing was performed.

Cortical thickness maps were calculated for each participant. Mean cortical thickness was measured and statistically compared using the Query Design Estimate Contrast (QDEC) tool, a single-binary application in FreeSurfer software, which identifies group differences. Analysis of cortical thickness was adjusted for age and gender, using a smoothing factor 
of 10. Free Surfer is hypothesis-free and can localize group differences in cortical thickness. All cortical regions were considered. Corrections for multiple comparisons in cortical thickness data were performed by the QDEC tool using Monte-Carlo simulation (significance set at $\mathrm{p}<0.05$ ), available in Free Surfer.

\section{Normality of thickness data distributions were tested using the Kolmogorov-Smirnov test.}

The data obtained from neuroimaging and clinical assessments were correlated and analyzed by Spearman's correlation coefficient, $\mathrm{p}<0.05$. Differences were tested using the Student's t-tests for paired samples. The Wilcoxon test was used for non-parametric statistics. Effect sizes (Cohen's) were included to demonstrate the magnitude of the difference between the migraine patients and controls. Effect sizes (Cohen's $d$ ) of approximately $0.2,0.4$ and 0.8 were considered to be small, medium and large effects, respectively. The Statistical Package for the Social Sciences 16.0 was used for this analysis.

\section{RESULTS}

Seven patients with migraine with aura and 12 patients with migraine without aura $(33.9 \pm 8.6$ years old, one male. According to IHS-III criteria: two with chronic migraine) were scanned pre- and post-treatment; and 19 matched healthy controls (33.1 \pm 9 years old, one male) were scanned once. The $\mathrm{HI}$ in the patients improved by $76.9 \%$ on average between the first and second scan (initial value: $159.8 \pm 125.5$; final value: $30.1 \pm 65.9 ; \mathrm{p}<0.001$ ) (Table 1 ).

\section{First scan vs. second scan comparisons in the patients}

The cortical thickness decreased by more than $1 \%$ in the second scan in nine (left hemisphere) and eight (right hemisphere) regions, and increased more than $1 \%$ in four (in each hemisphere) regions (Table 2).The areas in the left hemisphere with a significant cortical thickness decrease were: fusiform, lingual, posterior cingulate, and rostral middle frontal. Only in the left transverse temporal area did the thickness increase significantly. The areas in the right hemisphere with a significant thickness decrease were: caudal middle frontal, isthmus of cingulate, pars triangularis, posterior cingulate, rostral middle frontal and superior frontal. No cortical area showed a significant thickness increase on the right side. No thickness variation remained significant after the Monte Carlo corrections for multiple comparisons.

The HI variation correlated negatively with cortical thickness changes in different areas of the left hemisphere (Table 3). The left posterior cingulate thickness was the only region with a significant reduction in thickness after treatment and correlation (negative) with the HI.

\section{First scan vs. controls comparisons}

In the left hemisphere, the cortex was significantly thicker, in patients, only in the pars opercularis. The effect sizes were greater than 0.4 in the isthmus cingulate, paracentral, pericalcarine, post-central, posterior cingulate, precuneus, rostral anterior cingulate and superior parietal cortices. The thickness was significantly reduced in patients in the entorhinal cortex and temporal pole. The effect sizes were greater than 0.4 in the orbitofrontal, superior temporal and temporal pole cortices.

In the right hemisphere, the cortex was significantly thicker in patients in the paracentral, post-central, precuneus and superior parietal areas. The effect sizes were greater than 0.4 in the inferior parietal, isthmus cingulate, paracentral, pericalcarine, post-central, posterior cingulate, precentral, precuneus, superior parietal and supramarginal areas. The thickness was significantly reduced in patients in the entorhinal cortex. The effect sizes were greater than 0.4 in the pars orbitalis, temporal pole and insulacortices.

\section{Second scan vs. controls comparisons}

In the left hemisphere, the cortex was significantly thicker in patients in the paracentral and pars opercularis. The effect size was greater than 0.4 in the isthmus cingulate, paracentral, pars opercularis, pericalcarine, post-central, precuneus, rostral anterior cingulate, posterior cingulate, and superior parietal cortices. The thickness was significantly reduced in patients only in the entorhinal cortex. The effect sizes were greater than 0.4 in the entorhinal, parahippocampal, superior temporal and temporal pole cortices.

Table 1. Monthly headache characteristics before and after treatment.

\begin{tabular}{|c|c|c|c|c|c|c|c|}
\hline Variable & Treatment & Mean & SD & Min. & Median & Max. & $p$-value \\
\hline \multirow{2}{*}{ Headache frequency } & Pre-treatment & 6.6 & 4.2 & 1.0 & 5.0 & 16.0 & \multirow{2}{*}{$<0.001$} \\
\hline & Post-treatment & 2.0 & 2.2 & 0.0 & 1.0 & 8.0 & \\
\hline \multirow{2}{*}{ Attack duration } & Pre-treatment & 28.8 & 24.7 & 6.0 & 12.0 & 72.0 & \multirow{2}{*}{0.001} \\
\hline & Post-treatment & 12.0 & 17.5 & 0.0 & 6.0 & 72.0 & \\
\hline \multirow{2}{*}{ Headache index } & Pre-treatment & 159.8 & 125.5 & 8.0 & 144.0 & 384.0 & \multirow{2}{*}{$<0.001$} \\
\hline & Post-treatment & 30.1 & 65.9 & 0.0 & 8.0 & 288.0 & \\
\hline Percent HI improvement & & 76.9 & 31.1 & 0.0 & 87.5 & 100.0 & - \\
\hline
\end{tabular}

Frequency: headache days per month. Attack duration estimated in hours. Headache index (HI): frequency x duration. 
Table 2. Cortical thickness variation in the second scan

\begin{tabular}{|c|c|c|c|c|c|c|}
\hline \multirow{2}{*}{ Variable } & \multicolumn{3}{|c|}{ Left hemisphere } & \multicolumn{3}{|c|}{ Right hemisphere } \\
\hline & $\%$ Variation & Effect size & $p$-value & $\%$ Variation & Effect size & $p$-value \\
\hline Caudal anterior cingulate & -2.31 & -0.33 & 0.067 & -1.38 & -0.18 & 0.096 \\
\hline Caudal middle frontal & -1.11 & -0.28 & 0.058 & -1.55 & -0.38 & 0.013 \\
\hline Inferior temporal & -1.48 & -0.39 & 0.053 & - & - & - \\
\hline Isthmus of cingulate & - & - & - & -1.63 & -0.17 & 0.008 \\
\hline Lateral orbitofrontal & 2.24 & 0.21 & 0.253 & - & - & - \\
\hline Lingual & -1.45 & -0.30 & 0.003 & - & - & - \\
\hline Fusiform & $-1,52$ & $-0,33$ & 0,029 & - & - & - \\
\hline Medial orbitofrontal & 1.96 & 0.20 & 0.344 & - & - & - \\
\hline Parahippocampal & -1.04 & -0.11 & 0.310 & - & - & - \\
\hline Pars triangularis & - & - & - & -2.36 & -0.39 & 0.002 \\
\hline Posterior cingulate & -1.01 & -0.18 & 0.031 & -1.45 & -0.22 & 0.001 \\
\hline Rostral anterior cingulate & - & - & - & -1.56 & -0.17 & 0.363 \\
\hline Rostral middle frontal & -1.13 & -0.28 & 0.012 & -1.82 & -0.45 & 0.005 \\
\hline Superior frontal & - & - & - & -1.12 & -0.26 & 0.039 \\
\hline Frontal pole & -2.25 & -0.27 & 0.069 & 1.38 & 0.14 & 0.564 \\
\hline Temporal pole & 2.87 & 0.27 & 0.184 & 3.23 & 0.21 & 0.339 \\
\hline Transverse temporal & 1.72 & 0.22 & 0.012 & 1.75 & 0.43 & 0.091 \\
\hline Insula & - & - & - & 1.33 & 0.19 & 0.24 \\
\hline
\end{tabular}

Percent variations (increase or decrease) greater than $1 \%$ in cortical thickness at the second scan following prophylactic treatment. p-value : paired t- test. Effect size: Cohen's

Table 3. Correlations between \% headache index (HI) improvement and cortical thickness (second scan).

\begin{tabular}{lcc}
\hline Variable & rs & p-value \\
\hline Caudal middle frontal & -0.496 & 0.043 \\
Cuneus & -0.482 & 0.037 \\
Isthmus cingulate & -0.464 & 0.045 \\
Medial orbitofrontal & -0.515 & 0.024 \\
Paracentral & -0.533 & 0.019 \\
Posterior cingulate & -0.483 & 0.036 \\
Superior parietal & -0.514 & 0.024
\end{tabular}

All values correspond to the left hemisphere. No significant correlation was found at the right hemisphere. rs: Spearman's correlation coefficient.

In the right hemisphere, the cortex was significantly thicker in patients in the paracentral, post-central, precuneus and superior parietal areas. The effect size was greater than 0.4 in the isthmus cingulate, lingual, paracentral, pericalcarine, post-central, precentral, precuneus supramarginal and superior parietal areas.

\section{Monte-Carlo simulation}

In the patient group, there was no significant thickness variation comparing the second scan with the first scan. The Figure shows the cortical areas in the right hemisphere where the cortex is significantly thicker comparing the patients' first and second scans with controls.

\section{DISCUSSION}

This was one of the first attempts to correlate improvement of cortical thickness pre- and post-treatment in migraine. After multiple comparisons correction, only the right hemisphere showed thickness changes (increase), in patients vs. controls, located in the somatosensory and superior parietal cortices, both in the first and second scans. In addition, the right cortex was thicker in the second scan in the precentral, supramarginal, cuneus and precuneus when compared to controls. The effect size analysis showed small changes in various areas, specifically in the lingual, fusiform and caudal anterior cingulate cortices on the left (thickness reduction); and caudal middle frontal, pars triangularis, and rostral middle frontal (thickness reduction), and transverse temporal (thickness increase) cortices on the right. Patients improved significantly following treatment as shown by the $\mathrm{HI}$ reduction, but there were no corresponding changes in cortical thickness after correction for multiple comparison. Regression analysis showed significant negative correlations between the HI improvement and cortical thickness changes only in the left hemisphere.

The only area with significant thickness change (reduction, before Monte-Carlo correction) after treatment and simultaneous significant correlation (negative) with HI improvement in the second scan was the left posterior cingulate cortex. This region has been shown to be involved with nociception ${ }^{12}$. Pain has been shown experimentally to 


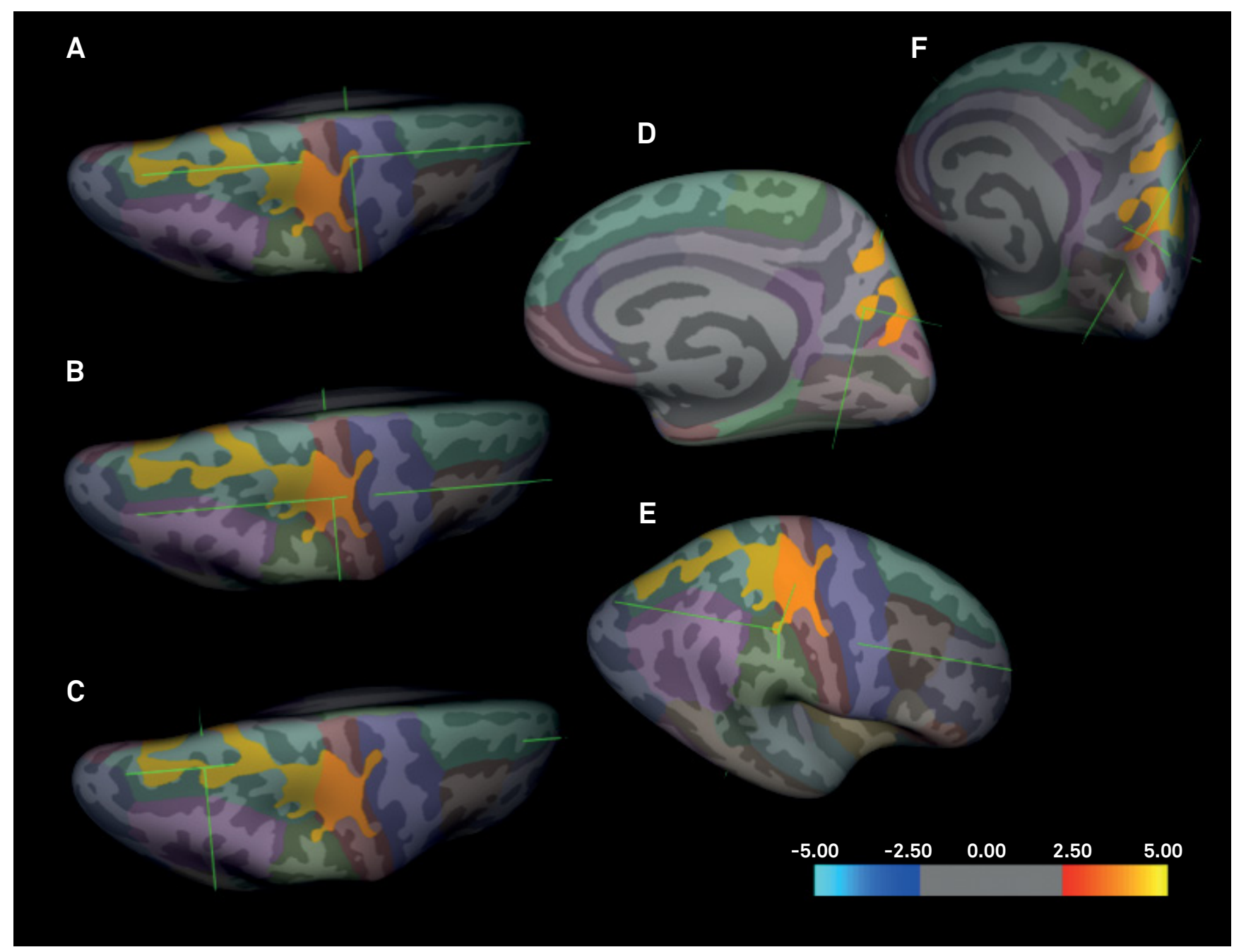

Figure. The figure shows cortical areas in the right hemisphere where the cortex is significantly thicker comparing the patients' first and second scans with controls. Regions of increased cortical thickness or surface area are shown in red (color-coded according to $t$ value), and regions of decreased cortical thickness or surface area are shown in blue (color-coded according to $t$ value). The color bar scale is logarithmic and represents - $\log 10$ (p): 2.5 corresponds to a p value of 0.05 and 5 corresponds to a p value of 0.00001 . Only clusters surviving multiple comparisons using the Monte Carlo simulation (10,000 permutations) are displayed. Cortical areas: A: Right precentral. B: Right post-central. C: Right superior parietal. D: Right precuneus. E: Right supramarginal. F: Right cuneus.

deactivate the posterior cingulate cortex ${ }^{13}$. This deactivation was reduced in patients with chronic pain ${ }^{14}$. The posterior cingulate cortex is part of the default mode network, a relevant area in pain physiology ${ }^{15}$. Reduced brain volumes in posterior cingulate cortex, a region reported to have antinociceptive functions, have been reported in various chronic pain conditions, such as phantom limb pain ${ }^{16}$, fibromyalgia $^{17}$, headache ${ }^{18}$ and trigeminal neuropathic pain ${ }^{19}$. In contrast, we did not find differences in posterior cingulate cortex thickness between the controls and patients. However, with our finding of decreased left posterior cingulate cortex thickness with $\mathrm{HI}$ improvement, we speculate that, in migraine, less antinociceptive activity in the left posterior cingulate cortex is required as the pain decreases.

In our study, the migraineurs showed a significant increase in cortical precuneus thickness in the right hemisphere when compared with controls. Maleki et al. reported that female migraine patients have thicker posterior insula and precuneus cortices compared with male migraineurs and healthy controls of both sexes ${ }^{20}$. The precuneus was shown to be more activated in females ${ }^{20}$, who are particularly susceptible to migraine. The anterior, central and posterior precuneus are involved with sensorimotor processing, cognition/associative processing, and visual processing, respectively. The three areas are functionally connected to the superior parietal cortex, paracentral lobule and motor cortex; the dorsolateral prefrontal, dorsomedial prefrontal and multimodal lateral inferior parietal cortex; and adjacent visual cortical regions ${ }^{21}$. Schwedt et al. also found negative correlations between pain thresholds and cortical thickness in the left posterior cingulate/precuneus in healthy individuals ${ }^{22}$. In contrast, migraineurs without aura exhibited positive correlations between pain thresholds and cortical thickness in the right precuneus ${ }^{22}$. Previous task-based fMRI studies have 
shown greater visual stimuli-induced precuneus activation in migraineurs without aura than in controls ${ }^{23}$. The changes found in the present and previous studies, associated with the fact that migraine patients may suffer visual, cognitive and sensorimotor deficits during attacks ${ }^{24}$, suggest that the cuneus is particularly dysfunctional in migraine.

In a previous report, migraineurs exhibited enhanced brain activation in the cerebellum anterior lobe/culmen, lingual gyrus, precuneus (all bilaterally), and the left cuneus while viewing negative pictures compared with neutral pictures $^{25}$. There are extensive connections between the precuneus and the dorsal premotor area, the supplementary motor area and the anterior cingulate cortex ${ }^{26}$. Compared with controls, migraineurs without aura showed a significant decrease in functional resting-state connectivity between the left precuneus and the left inferior and superior occipital gyrus, bilateral middle occipital gyrus, bilateral cuneus, bilateral superior parietal lobules, bilateral somatosensory cortex, bilateral dorsolateral prefrontal cortices, right premotor cortex, pons, bilateral cerebellar posterior lobes, right paracentral lobule, right middle cingulate gyrus and bilateral supplementary motor areas ${ }^{27}$. All these brain regions are involved with pain processing ${ }^{23}$.

The somatosensory cortex has been shown to be thicker in migraine patients, which is in line with the present findings. Activation of the somatosensory cortices has been reported in approximately $75 \%$ of human imaging studies of pain ${ }^{28}$. This area is strongly implicated in the ascending trigemino-cortical nociceptive pathway. Thickening of the somatosensory cortex has been demonstrated in migraine ${ }^{6,29}$. Migraine patients with a higher headache frequency (8-14 days/month) showed increased post-central gyrus thickness in comparison with low frequency (less than two days/months) patients ${ }^{3}$. Kim et al. compared migraine patients with controls and found that migraine patients had cortical thickening in bilateral postcentral gyrus $^{30}$. Whether these abnormalities contribute to migraine susceptibility or, conversely, are the consequence of repeated attacks, is still a matter of debate.

Our results showed that migraineurs presented with cortical thickening in two areas of the parietal lobe (superior parietal lobe and supramarginal gyrus). The inferior parietal lobe is mainly involved in top-down control of executive functions and in cognitive aspects of processing sensory stimuli, including pain ${ }^{31}$. Migraine with aura patients showed bilateral thickening of regions in the inferior parietal lobe ${ }^{32}$, in contrast with another study ${ }^{33}$.

Although several studies, including ours, confirm cortical thickness changes in migraine, a larger patient cohort failed to replicate cortical thickness findings in both migraine patients with, and without, aura relative to controls ${ }^{5}$. Methodological issues are probably the best explanation for those discrepancies.

Our study confirms that neuroimaging may demonstrate changes in cortical areas previously shown to be involved with the lateral pain system. That system consists of neurons of the spinothalamic tract that project to the somatosensory nuclei of thalamus, which in turn transfer nociceptive information to the primary and secondary somatosensory areas, and posterior insula; these discriminatory areas determine the localization, the intensity, and the quality of pain and are connected to the parietal cortex ${ }^{34}$.

Most of our findings were lateralized. Since our patients presented with bilateral ( five individuals), alternating (nine), predominantly left (four), or right (one) pain, the laterality of our data cannot be explained by headache side preference, even though predominantly left-sided headache was more frequent. Bilateral reduction in regional cerebral blood flow has been documented in the cingulofrontal transitional cortex and posterior cingulate cortex during noxious stimulation of the left hand ${ }^{35}$. Similar to our results, in patients with HIV-associated distal neuropathic pain, changes (increase) were restricted to the left posterior cingulate cortex ${ }^{36}$. A negative correlation between pain thresholds and cortical thickness was also reported on the left ${ }^{22}$. Further data with larger samples are required for better understanding of the laterality findings.

The potential reversibility of cortical changes following treatment suggested in this study raises multiple questions. Firstly, it remains to be determined what the pathological bases of the thickness changes are, and how migraine triggers the tissue adaptations ${ }^{4}$. Secondly, what are the mechanisms responsible for the return to previous thickness, an effect probably related to headache frequency and duration? Thirdly, what are the implications of these changes in cortical spreading depression? Fourthly, how could these changes, either isolated or in combination with other neuroimaging findings, eventually serve as biomarkers for diagnosis, severity, or drug efficacy?

Cortical thickness changes occur because of hypo- or hyper-function secondary to local, near or distant connections $^{37}$. Although treatment can theoretically change cortical thickness in affected regions, the present results do not allow causation conclusions. Our study has strong aspects. All patients were treatment naïve at the first scan; they were examined by the same physicians under a unique protocol and usual care, reproducing real life treatment; and the observation was over a long period of time, on average. There are many weak points to be considered. Firstly, the time span between the first and second scan in our patients varied considerably. Secondly, due to the study length, patients did not record a precise headache diary, leading to potential recall biases. Thirdly, the sample size was relatively small. Fourthly, controls were not scanned twice, at the same time as the patients. Finally, although we are aware that cortical thickness may be affected by fortuitous influences, to the best of our knowledge, no confounding factors influenced the results presented herein. Nevertheless, the significant correlations between the HI percentage variation and cortical thickness 
reduction in regions known to be affected in migraineurs, point to the hypothesis that anatomical variations induced by the disease are not irreversible and allow the hypothesis that treatment may reverse cortical thickness modifications in migraine.

Taken together, the data show that there are differences in cortical thickness between migraine patients and controls. Among the regions with significant change in cortical thickness following preventive treatment, the degree of improvement correlated with reduction in thickness in the left posterior cingulate. Previous studies have shown that this area is involved with pain processing and is affected in headache disorders. To investigate the reasons for intriguing right-to-left changes and to clarify controversies still remaining in this field, young migraine-free subjects with migraine parents should be scanned at base-line and re-examined later in the event of migraine development.

\section{References}

1. Woldeamanuel YW, Cowan RP. Migraine affects 1 in 10 people worldwide featuring recent rise: A systematic review and meta-analysis of community-based studies involving 6 million participants. J Neurol Sci. 2017 Jan;372:307-15. https://doi.org/10.1016/j.jns.2016.11.071

2. Burstein R, Noseda R, Borsook D. Migraine: multiple processes, complex pathophysiology. J Neurosci. 2015 Apr;35(17):6619-29. https://doi.org/10.1523/JNEUROSCI.0373-15.2015

3. Maleki N, Becerra L, Brawn J, Bigal M, Burstein R, Borsook D. Concurrent functional and structural cortical alterations in migraine. Cephalalgia. 2012 Jun;32(8):607-20. https://doi.org/10.1177/0333102412445622

4. Hadjikhani N. Relevance of cortical thickness in migraine sufferers. Expert Rev Neurother. 2008 Mar;8(3):327-9. https://doi.org/10.1586/14737175.8.3.327

5. Datta R, Detre JA, Aguirre GK, Cucchiara B. Absence of changes in cortical thickness in patients with migraine. Cephalalgia. 2011 Oct;31(14):1452-8. https://doi.org/10.1177/0333102411421025

6. DaSilva AF, Granziera C, Snyder J, Hadjikhani N. Thickening in the somatosensory cortex of patients with migraine. Neurology. 2007 Nov;69(21):1990-5. https://doi.org/10.1212/01.wnl.0000291618.32247.2d

7. Maleki N, Becerra L, Nutile L, Pendse G, Brawn J, Bigal M et al. Migraine attacks the Basal Ganglia. Mol Pain. 2011 Sep;7:71. https://doi.org/10.1186/1744-8069-7-71

8. Schwedt TJ, Dodick DW. Advanced neuroimaging of migraine. Lancet Neurol. 2009 Jun;8(6):560-8. https://doi.org/10.1016/S1474-4422(09)70107-3

9. Lipton RB, Bigal ME, Scher AI, Stewart WF. The global burden of migraine. J Headache Pain. 2003 Mar;4 Suppl 1:s3-11. https://doi.org/10.1007/s101940300001

10. Headache Classification Committee of the International Headache S. The International Classification of Headache Disorders. 2nd edition. Cephalalgia. 2004;24(1 Suppl):9-160.

11. Headache Classification Committee of the International Headache S. The International Classification of Headache Disorders. 3rd edition (beta version). Cephalalgia. 2013;33(9):629-808. https://doi.org/10.1177/0333102413485658

12. Emerson NM,Zeidan F, Lobanov OV, Hadsel MS, Martucci KT, Quevedo AS et al. Pain sensitivity is inversely related to regional grey matter density in the brain. Pain. 2014;155(3):566-73). https://doi.org/10.1016/j.pain.2013.12.004

13. Kong J, Loggia ML, Zyloney C, Tu P, Laviolette P, Gollub RL. Exploring the brain in pain: activations, deactivations and their relation. Pain. 2010;148(2):257-67). https://doi.org/10.1016/j.pain.2009.11.008

14. Baliki MN, Geha PY, Apkarian AV, Chialvo DR. Beyond feeling: chronic pain hurts the brain, disrupting the default-mode network dynamics. J Neurosci. 2008;28(6):1398-403). https://doi.org/10.1523/JNEUROSCI.4123-07.2008

15. Raichle ME, MacLeod AM, Snyder AZ, Powers WJ, Gusnard DA, Shulman GL. A default mode of brain function. Proc Natl Acad Sci U S A. 2001;98(2):676-82). https://doi.org/10.1073/pnas.98.2.676

16. Draganski B, Moser T, Lummel N, Gänssbauer S, Bogdahn $U$, Haas F et al. Decrease of thalamic gray matter following limb amputation. Neuroimage. 2006;31(3):951-7). https://doi.org/10.1016/j.neuroimage.2006.01.018

17. Robinson ME, Craggs JG, Price DD, Perlstein WM, Staud R. Gray matter volumes of pain-related brain areas are decreased in fibromyalgia syndrome. J Pain. 2011;12(4):436-43). https://doi.org/10.1016/j.jpain.2010.10.003

18. Schmidt-Wilcke T, Leinisch E, Straube A, Kämpfe N, Draganski $\mathrm{B}$, Diener $\mathrm{HC}$ et al. Gray matter decrease in patients with chronic tension type headache. Neurology. 2005;65(9):1483-6). https://doi.org/10.1212/01.wnl.0000183067.94400.80

19. DaSilva AF, Becerra L, Pendse G, Chizh B, Tully S, Borsook D. Colocalized structural and functional changes in the cortex of patients with trigeminal neuropathic pain. PLoS One. 2008;3(10):e3396. https://doi.org/10.1371/journal.pone.0003396

20. Maleki N, Linnman C, Brawn J, Burstein R, Becerra L, Borsook D. Her versus his migraine: multiple sex differences in brain function and structure. Brain. 2012 Aug;135(Pt 8):2546-59. https://doi.org/10.1093/brain/aws175

21. Margulies DS, Vincent JL, Kelly C, Lohmann G, Uddin LQ, Biswal BB et al. Precuneus shares intrinsic functional architecture in humans and monkeys. Proc Natl Acad Sci USA. 2009 Nov;106(47):20069-74. https://doi.org/10.1073/pnas.0905314106

22. Schwedt TJ, Chong CD. Correlations between brain cortical thickness and cutaneous pain thresholds are atypical in adults with migraine. PLoS One. 2014Jun;9(6):e99791. https://doi.org/10.1371/journal.pone.0099791

23. Schwedt TJ, Chiang CC, Chong CD, Dodick DW. Functional MRI of migraine. Lancet Neurol. 2015 Jan;14(1):81-91. https://doi.org/10.1016/S1474-4422(14)70193-0

24. Vincent MB, Hadjikhani N. Migraine aura and related phenomena: beyond scotomata and scintillations. Cephalalgia. 2007 Dec;27(12):1368-77. https://doi.org/10.1111/j.1468-2982.2007.01388.x

25. Wang M, Su J, Zhang J, Zhao Y, Yao Q, Zhang Q et al. Visual cortex and cerebellum hyperactivation during negative emotion picture stimuli in migraine patients. Sci Rep. 2017 Feb;7:41919. https://doi.org/10.1038/srep41919

26. Petrides M, Pandya DN. Projections to the frontal cortex from the posterior parietal region in the rhesus monkey.J Comp Neurol. 1984 Sep;228(1):105-16. https://doi.org/10.1002/cne.902280110

27. Zhang J, Su J, Wang M, Zhao Y, Yao Q, Zhang Q et al. Increased default mode network connectivity and increased regional homogeneity in migraineurs without aura. J Headache Pain. 2016 Dec;17(1):98. https://doi.org/10.1186/s10194-016-0692-z

28. Apkarian AV, Bushnell MC, Treede RD, Zubieta JK. Human brain mechanisms of pain perception and regulation in health and disease. Eur J Pain. 2005 Aug;9(4):463-84. https://doi.org/10.1016/j.ejpain.2004.11.001

29. Chong CD, Starling AJ, Schwedt TJ. Interictal photosensitivity associates with altered brain structure in patients with episodic migraine. Cephalalgia. 2016 May;36(6):526-33. https://doi.org/10.1177/0333102415606080 
30. Kim JH, Kim JB, Suh SI, Seo WK, Oh K, Koh SB. Thickening of the somatosensory cortex in migraine without aura. Cephalalgia: an international journal of headache. 2014;34(14):1125-33. https://doi.org/10.1177/0333102414531155

31. Weidner R, Krummenacher J, Reimann B, Müller HJ, Fink GR. Sources of top-down control in visual search.J Cogn Neurosci. 2009 Nov;21(11):2100-13. https://doi.org/10.1162/jocn.2008.21173

32. Messina R, Rocca MA, Colombo B, Valsasina P, Horsfield MA, Copetti $\mathrm{M}$ et al. Cortical abnormalities in patients with migraine: a surface-based analysis. Radiology. 2013 Jul;268(1):170-80. https://doi.org/10.1148/radiol.13122004

33. Schmitz N, Arkink EB, Mulder M, Rubia K, Admiraal-Behloul F, Schoonman GG et al. Frontal lobe structure and executive function in migraine patients. Neurosci Lett. 2008 Aug 1;440(2):92-96. https://doi.org/10.1016/j.neulet.2008.05.033
34. Grazzi L, Chiapparini L, Ferraro S, Usai S, Andrasik F, Mandelli ML et al. Chronic migraine with medication overuse pre-post withdrawal of symptomatic medication: clinical results and fMRI correlations. Headache. 2010 Jun;50(6):998-1004. https://doi.org/10.1111/j.1526-4610.2010.01695.x

35. Vogt BA, Derbyshire S, Jones AK. Pain processing in four regions of human cingulate cortex localized with co-registered PET and MR imaging. Eur J Neurosci. 1996;8(7):1461-73). https://doi.org/10.1111/j.1460-9568.1996.tb01608.x

36. Keltner JR, Connolly CG, Vaida F, Jenkinson M, Fennema-Notestine C, Archibald S et al HIV Distal Neuropathic Pain Is Associated with smaller ventral posterior cingulate cortex. Pain Med. 2017 Mar;18(3):428-40. https://doi.org/10.1093/pm/pnw180

37. Catani M, Ffytche $\mathrm{DH}$. The rises and falls of disconnection syndromes. Brain. 2005 Oct;128(Pt 10):2224-39. https://doi.org/10.1093/brain/awh622 